\title{
Scrivere di storia contemporanea su Wikipedia. Una proposta per un laboratorio on line
}

\section{di Elena Mastretta}

Mar 3, 2021 | Didattica in classe, In evidenza | $\underline{0} \mid$

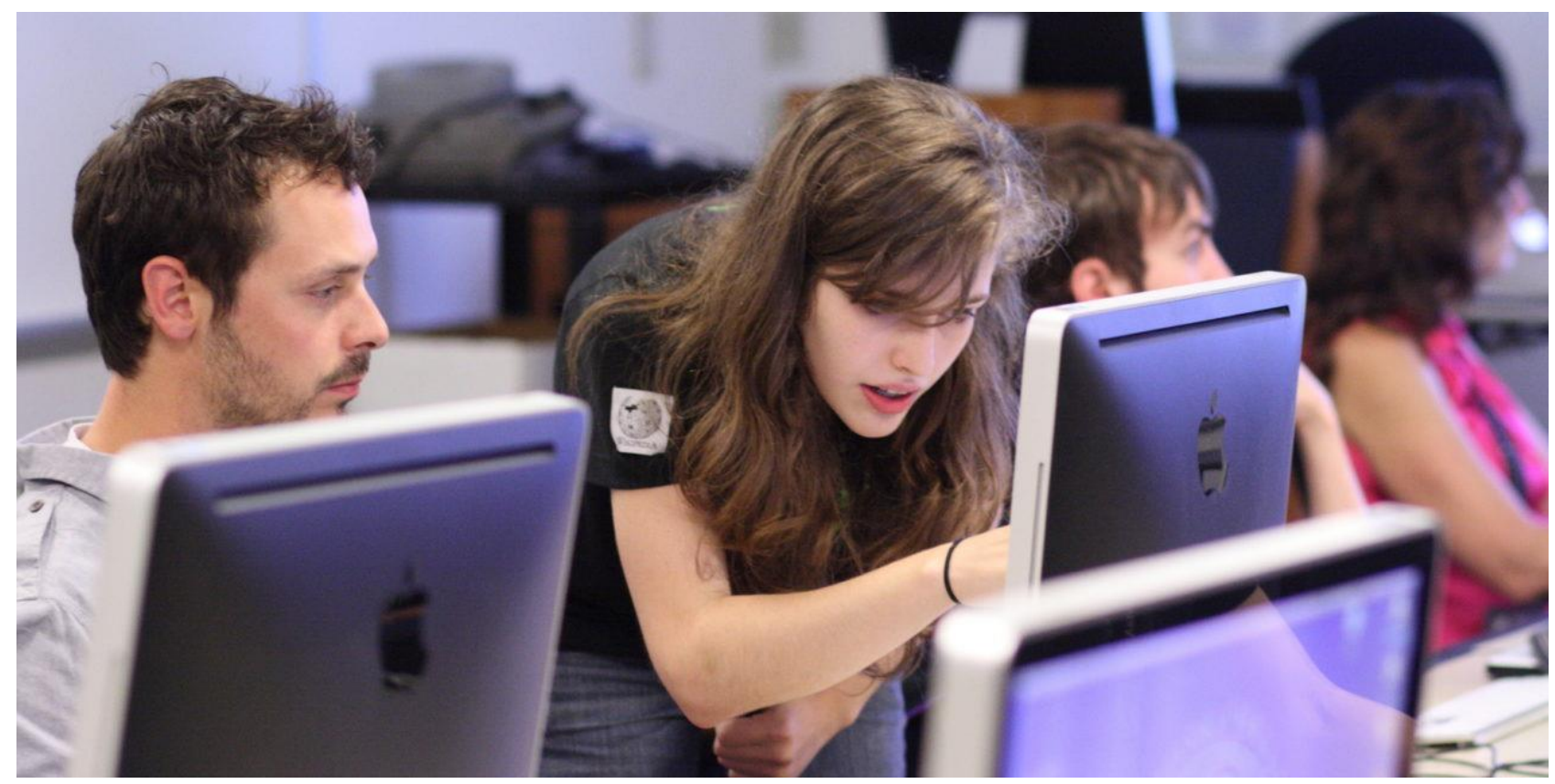

Participanti all'incontro "Wikipedia in Higher Education" a Boston, Massachusetts, 7-9 Luglio 2011.

Foto di Sage Ross - Own work, CC BY-SA 3.0, $\underline{\text { Link }}$

\section{Abstract}

Laboratorio di storia realizzato in forma online nel corso della Summer School della rete nazionale edizione 2020. L'attività è stata strutturata per evidenziare le potenzialità didattiche insite nel processo di scrittura di voci di Wikipedia e mostra cos'è e come si usa l'enciclopedia libera, quali sono le differenti modalità per collaborare e contribuire al progetto attraverso esemplificazioni dell'analisi delle fonti, della stesura collettiva di testi informativi a partire dall'esperienza di docenti che hanno lavoro realizzato con la classe negli ultimi tre anni.

Wikipedia è un'enciclopedia libera online d'innegabile importanza nel mondo di oggi e con una storia interessante alle sue spalle: il numero di utenti, di consultazioni e di versioni (noi siamo abituati ad usare Wikipedia in italiano, ma esistono centinaia di lingue di scrittura e, in alcuni casi, la sua compilazione è l'unica modalità non orale di trasmissione di quell'idioma e della cultura ad esso correlata) corrispondono a cifre sbalorditive, che ci impediscono di ignorarne l'esistenza. Iniziare a esserne scrittore di voci permette anche di comprendere che, sebbene i contenuti siano compilati da volontari e quindi la loro correttezza possa essere ancora imperfetta, esistono regole abbastanza rigide per la stesura.

Le voci di storia contemporanea non sono molto presenti nelle diverse versioni linguistiche, 
soprattutto perché Wikipedia non è un luogo dove pubblicare inediti, ma un'enciclopedia, ossia una fonte terziaria che non usa fonti primarie, bensì secondarie.

Quali sono dunque le regole cui deve attenersi chi inizia la scrittura di voci? Le si trovano chiarite nei cosiddetti "cinque pilastri".

\section{I cinque pilastri}

Primo pilastro: Wikipedia è un'enciclopedia

Asserisce il principio di enciclopedicità delle voci inserite: non tutto trova posto su Wikipedia. Quando, tra il 2016 e il 2017, la rete degli istituti storici piemontesi coordinata dall'Istoreto inserì nei già attivi percorsi TIC e didattica della storia l'uso di Wikipedia, il problema dell'enciclopedicità delle voci di storia contemporanea, in particolar modo di quella locale, si pose immediatamente e la storia della costruzione della voce di Remo Jona è, a mio avviso il miglior esempio di come i due sguardi e le due posizioni, quelli degli storici e quelli degli enciclopedisti, hanno finito per convergere, anche grazie a una serie di iniziative tutte a declinazione didattica, simili a quella fatta nel workshop della Summer School 2020, che hanno visto infittirsi la collaborazione tra la rete degli Istituti Storici e la struttura italiana di Wikipedia, in un primo tempo, quindi con l'associazione InformAzionI successivamente: parlandosi e confrontandosi si è riusciti a comprendere le diversità di approccio, motivati dal comune interesse per la crescita dell'enciclopedia libera anche su questi temi con voci che fossero di rilievo e corrette[1].

Secondo pilastro: Wikipedia ha un punto di vista neutrale

Ossia: la storia si basa sui documenti prima che sulle interpretazioni. Nel caso della ricostruzione di un fatto storico all'interno di Wikipedia, non ci baseremo direttamente su fonti primarie, bensì secondarie.

Se provate a cercare delle voci sull'enciclopedia libera, potreste imbattervi in un avviso di questo tipo:

La neutralità di questa voce o sezione sull'argomento storia contemporanea è stata messa in dubbio.

Motivo: la voce in più punti è apertamente schierata e presenta opinioni non referenziate e "nicerche originali", da rivedere.

Per contribuire, correggi i toni enfatici o di parte e partecipa alla discussione. Non rimuovere questo avviso finché la disputa non è risolta. Segui i suggerimenti del progetto di riferimento

Questa voce o sezione sull'argomento storia contemporanea è ritenuta da controllare.

Motivo: La voce è frutto di una visione distorta e pretestuosa della storia ed è infarcita di considerazioni al limite della corbelleria. Da rifare

Partecipa alla discussione e/o correggi la voce. Segui i suggerimenti del progetto di riferimento

Compito di una enciclopedia è di raccogliere voci informative. I fatti di storia contemporanea, e non solo quelli, possono avere più interpretazioni. Dovere di chi stende la voce è quello di fornire una visione neutrale dei fatti e dello stato di conoscenza su quell'argomento, portando, se esistono, $\mathrm{i}$ documenti relativi a tutte le possibili interpretazioni esistenti qualora non ve ne fosse una univocamente accettata. Non è ammesso fornire una versione a favore del proprio punto di vista né ignorare quello altrui.

Terzo pilastro: Wikipedia è libera

Nelle vostre incursioni su Wikipedia potreste avere visto un banner che invita a fare una donazione al progetto. Le cifre raccolte servono a finanziare il progetto, in particolar modo l'infrastruttura hardware necessaria a permetterne l'utilizzo su scala mondiale. Lo stesso scopo potrebbe essere raggiungo accettando di inserire banner pubblicitari: scelta, però, che si scontrerebbe con la neutralità di quanto pubblicato e quindi non percorsa. Questo però non è l'unico senso in cui si dice che Wikipedia è libera: libera è la partecipazione (chiunque rispetti le regole può contribuire) e i contenuti sono pubblicati e rilasciati secondo particolari licenze (CC BY-SA e GFDL), che vogliono far diventare realtà il sogno illuminista di un sapere universale per tutti. 
Quarto pilastro: Wikipedia ha un codice di condotta

Il fatto che il contributo di tutti sia ben accetto non significa che le modifiche alle voci presenti possano essere fatte indiscriminatamente. L'utente che si avvicina a Wikipedia deve ricordarsi che si tratta di un progetto collaborativo e che tutte le modifiche sono registrate, anche quelle fatte dagli "utenti anonimi". Per la salvaguardia dei suoi contenuti Wikipedia registra infatti gli indirizzi IP di chi contribuisce. Se pensiamo di poter migliorare una voce, nell'interesse di tutti, ricordiamoci che esistono delle pagine di discussione: cancellare e riscrivere, anche se viene consigliato di non essere timidi nel portare il proprio contributo, non è sempre la scelta migliore. Allo stesso modo, se ci sentiamo danneggiati dalla modifica che altri hanno fatto a ciò che abbiamo scritto noi, discutiamone negli appositi spazi. Wikipedia è anche una comunità e farne parte può rivelarsi piacevole, oltre che educativo.

Quinto pilastro: Wikipedia non ha regole fisse...

Fatti salvi i cinque pilastri, spesso i nuovi utenti sono spaventati dalla formattazione delle voci, dalla imperfezione che caratterizza le prime stesure, temono di essere mal giudicati. Nulla di tutto ciò. Un progetto collaborativo basato sul volontariato si giova dell'apporto di tutti. Quando vi registrerete, e continuando a leggere capirete perché è meglio agire da utente registrato, verrete accolti nella comunità con lo spirito Wikilove. Poiché tutte le versioni delle voci vengono salvate, non è possibile fare danni irreparabili. Meglio una voce imperfetta che una voce assente.

\section{Cosa fare per cominciare?}

Al contrario di quanto avviene sui social network - dove è il primo passaggio - registrarsi non è indispensabile per leggere e produrre contenuti (benché rimanga un'operazione importante). Approcciandoci a questo mondo, dobbiamo innanzi tutto chiederci su quale voce possiamo agire con modifiche/integrazioni piuttosto che con una creazione ex novo. La consultazione e anche le modifiche, infatti, possono essere fatte senza avere un account. Come abbiamo già sottolineato, viene tenuta traccia anche dei contributi apportati da utenti non registrati. Quando si decide di costruire un percorso scolastico intorno alla creazione di voci, però, una volta definito ciò su cui si vuole agire (4-5 voci sono un numero sufficiente per un gruppo classe medio da realizzarsi in un progetto annuale), bisogna procurarsi una buona bibliografia, suddividere i ragazzi in piccoli gruppi, distribuire loro dei compiti precisi e, a quel punto, bisogna fare in modo che ciascuno diventi un utente registrato di Wikipedia: insomma, la premessa non è diversa da quella di una qualunque ricerca in ambito storico.

Attenzione, poi: se state lavorando da scuola, utilizzando un solo indirizzo IP non potrete far registrare tutti gli studenti nello stesso momento, pena il blocco temporaneo del sistema, che riconosce il numero massiccio di iscrizioni come un tentativo di violazione. La cosa migliore è che $\mathrm{i}$ ragazzi arrivino già con un account creato, di cui vi avranno comunicato il nikname. Ricordate loro di memorizzare l'indirizzo email che utilizzano al momento dell'iscrizione. Solo attraverso la mail, in caso di dimenticanza, è possibile recuperare la password: nemmeno gli amministratori possono fare questa operazione. Anche se lavoreranno a gruppi su una stessa voce, non si devono usare account collettivi. Ciascuno deve avere il suo sia per correttezza di utilizzo dell'ambiente, sia per permettere all'insegnante di tracciare il lavoro dei singoli alunni. 
Come insegnanti, il vantaggio di avere studenti che contribuiscono alla scrittura di voci registrate è quello di poter facilmente individuare, tramite i nikname, chi ha fatto cosa e quando. Soprattutto qualora si decida, seguendo il nostro consiglio, di far lavorare i ragazzi a piccoli gruppi, il poter controllare la stesura riduce molte incertezze sulla valutazione sul lavoro svolto. Gli utenti registrati hanno comunque più opportunità rispetto a quelli anonimi: come riportato nella pagina "perché registrarsi?" (https://it.wikipedia.org/wiki/Aiuto:Login\#Perch\%C3\%A9_farlo?) essi

- possono tenere sotto controllo le voci a cui sono interessati attraverso la propria lista di "osservati speciali",

- possono contrassegnare una modifica come "minore", rendendo più facile il lavoro di controllo degli altri utenti;

- dispongono di una pagina utente personale, nella quale è possibile inserire alcune informazioni sui propri interessi enciclopedici, e di una propria pagina di discussione, utile per comunicare direttamente con gli altri utenti;

- possono navigare ed interagire con i siti WMF completamente in HTTPS, in automatico: è uno dei vantaggi della registrazione

- navigano e interagiscono con i siti WMF completamente in HTTPS, con sensibile vantaggio in termini di sicurezza e privacy.

Qualora si proseguisse nella compilazione, ecco gli altri vantaggi:

A ciò si aggiunga che, dopo dieci giorni dalla registrazione e cinquanta modifiche effettuate, si diventa utenti autoconvalidati. Ciò permette di:

- $\quad$ Spostare pagine (cioè rinominarle)

- Inserire collegamenti esterni senza bisogno di inserire il CAPTCHA

- Modificare pagine parzialmente protette

- Caricare e sovrascrivere immagini e altri file multimediali

\section{Come si inizia a scrivere?}

Una volta individuata la voce e stesa la bibliografia minima indispensabile[2], il processo di scrittura all'inizio è simile a quello che servirebbe per compilare un testo informativo cartaceo. Se è lecito copiare i contenuti di Wikipedia all' esterno di essa citando la fonte, non è possibile inserire contenuti non rielaborati nelle voci che si stanno costruendo. Quando un ragazzo, o un gruppo di ragazzi, ha individuato nelle fonti a sua disposizione gli elementi che vuole riportare nella voce, deve procedere con una rielaborazione che abbia come esito un testo nuovo rispetto a quelli di partenza. Nel caso in cui chi sta scrivendo la voce sia l'estensore di un testo "perfetto" su quello stesso argomento e non voglia modificarlo, è possibile riportarlo in Wikipedia dopo avere compilato un'apposita richiesta che dà origine ad un Tiket OTRS. Questa procedura, come molte altre che servono per scrivere bene una voce, è spiegata nelle pagine di aiuto di Wikipedia.

\section{La sandbox}

Un buono strumento per la creazione collaborativa delle voci è la sandbox o pagina delle prove. "Sabbionaia" in inglese, è uno spazio dove è possibile effettuare prove per impratichirsi con Wikipedia e il software MediaWiki, in tutta sicurezza e libertà. Il link della sandbox può essere 
condiviso e più utenti possono lavorarci. Gli utenti registrati possono creare pagine di prova personalizzate, una per ogni progetto. E' importante, nel caso di un lavoro che si protrae nel tempo, avere una sandbox personale: quella a disposizione di tutti gli utenti viene periodicamente sovrascritta.

\section{Note e bibliografia}

Ogni dato inserito deve essere verificabile, quindi se si cita una data, un nome, in nota va riportata la fonte. La compilazione delle note è particolarmente importante e deve essere curata. Infine, si inseriscono bibliografia e sitografia, collegamenti esterni. Quello delle note e della bibliografia è un aspetto cui non sempre i ragazzi prestano la dovuta attenzione, ma permette loro di acquisire una competenza importante nella stesura di testi.

\section{Qualche riflessione teorica sul progetto}

Come abbiamo avuto modo di verificare anche nel corso del workshop della Summer 2020, al di là dei tecnicismi che bisogna acquisire per inserire il proprio testo nell'enciclopedia libera, un lavoro su Wikipedia implica, rispetto ad altri, una riflessione teorica abbastanza elevata per un compito laboratoriale. Si tratta di un mondo verso il quale il corpo docente ha sempre una grande diffidenza, vuoi per la mancanza di autorialità, vuoi per la facilità con cui i ragazzi ne estraggono "soluzioni pronte" ai compiti che assegniamo loro.

\section{Il laboratorio della Summer}

Anche per entrare nel vivo del ragionamento e per mettere i docenti nella stessa condizione in cui si troveranno i loro studenti, nella Summer 2020 abbiamo proposto ai partecipanti divisi in piccoli gruppi di lavorare su voci esistenti, ma problematiche. Essendo il contenuto del workshop strettamente legato a quello della conferenza del giorno precedente, si è lavorato sulla coppia uguaglianza/disuguaglianza prendendo in esame voci come razzismo, tolleranza, ecc. La consegna era quella di immaginare un percorso didattico finalizzato al perfezionamento di quella voce da stendersi anche con l'aiuto di una griglia consegnata in precedenza.

Dopo una presentazione delle caratteristiche dell'enciclopedia libera sono stati proposte 2 videotestimonianze di docenti che avevano realizzato il percorso nelle loro classi nell'anno scolastico appena concluso.

I partecipanti al laboratorio avevano a disposizione circa 30 minuti ed erano stati invitati a crearsi un'utenza precedentemente. Il tempo è stato quasi sempre utilizzato per analizzare le fonti messe a disposizione (fonti in rete) e per pensare a quali cambiamenti avrebbero potuto essere apportati alla voce assegnata non nel tempo del laboratorio, assolutamente non sufficiente, ma nell'UDA che erano invitati a co-progettare per le loro classi sulla base del modello fornito. Ogni gruppo di quattro insegnanti è stato composto tenendo conto del doppio fattore geografico e dell'ordine di scuola. Al termine della sessione in piccolo gruppo si è tornati in plenaria per discutere dei progetti proposti e/o delle difficoltà incontrate. 
Da subito, sebbene i docenti fossero stati avvisati del fatto che dalla nostra esperienza avevamo compreso come fosse più difficile modificare una voce esistente piuttosto che crearne una ex novo[3], l'ampiezza di alcune voci ha creato disorientamento. Sono state così suggerite alcune strategie, come quella di provare a sotto-tematizzare l'argomento. Si tratta di un metodo di lavoro che negli anni ci ha dato buoni risultati: come un argomento complesso può essere ben affrontato con uno studio di caso, così, se mettere mano alla voce razzismo può sembrare un compito non realizzabile, possiamo cercare una biografia, un luogo/monumento, un evento, magari riferibili alla nostra realtà locale sul tema del razzismo da far diventare voce di Wikipedia, e da qui il discorso può essere poi allargato.

Nella discussione in plenaria un portavoce per ciascun gruppo ha illustrato la piccola progettazione realizzata e si sono poi affrontate le domande dei corsisti relative alle caratteristiche di Wikipedia. La Summer School 2020 non è terminata con la fase dei laboratori: il progetto prevede il tutoraggio per chi desidera sperimentare il laboratorio frequentato nelle proprie classi e così è stato anche per quello su Wikipedia, per il quale al momento ogni corista sta compulando una diversa voce biografica.

Il motivo per cui abbiamo scelto di dedicare molta parte della formazione docenti al tema delle TIC e in particolare a Wikipedia, proponendolo anche nella scuola estiva, sta nelle potenzialità che questo ambiente offre a livello didattico: la didattica cooperativa, il controllo sul lavoro dei singoli ragazzi, la trasversalità che può essere creata attraverso un'accurata scelta delle voci da compilare, l'educazione civica sperimentata nel rispetto dei cinque pilastri e del wikilove. Inutile, soprattutto in un momento come quello che stiamo vivendo, sottolineare i vantaggi di un percorso didattico che può essere fruito a distanza in modo collaborativo.

\section{Stimoli per lavorare in classe}

Mettiamo a disposizione di tutti i lettori di Novecento.org i due video utilizzati nel nostro laboratorio e realizzati da due docenti del cuneese che hanno svolto il percorso con la loro classe nell'a.s. 2019/20[4]. I colleghi hanno riassunto le fasi del loro lavoro evidenziandone punti di forza e di debolezza e la loro viva voce potrà fugare le ultime perplessità di chi ancora ha dubbi sull'intraprendere l'attività. Alleghiamo anche la scheda di osservazione propostz per la valutazione del progetto e un esempio di costruzione di macroarea sui temi proposti dalla Summer School, che possono servire come traccia per la proposta di un percorso, che immaginiamo di educazione civica, avente come compito di realtà la creazione di una voce di Wikipedia.

Note:

[1] Molte osservazioni e considerazioni sono contenute negli articoli di novecento.org firmati proprio di Flavio Febbraro, che vi invitiamo a leggere. 
[2] Wikipedia è una fonte terziaria che utilizza fonti secondarie. La buona regola sarebbe quella di avere per ogni nuova voce almeno tre titoli pubblicati. Sono ammesse anche le fonti on line, se hanno una certa verificabilità.

[3] Questo aspetto era stato in particolare al centro del progetto Wikipedia dell'a.s. 2018/19 ed è quello su cui sono emerse le maggiori difficoltà nel portare a termine le voci. Purtroppo, la prematura scomparsa di Flavio Febbraro, che lo conduceva, ci ha poi impedito di fare, ad oggi, tutte le riflessioni necessarie per sciogliere questo nodo.

[4] I percorsi sono stati curati dall'Istituto Storico Livio Bianco di Cuneo, in particolare dal collega Gigi Garelli che è stato con me tutor di questo laboratorio nella Summer School 2020. 